\title{
Publisher's Note: Validity of the empty-beam correction in near-field imaging [Phys. Rev. A 91, 013821 (2015)]
}

\author{
C. Homann, T. Hohage, J. Hagemann, A.-L. Robisch, and T. Salditt \\ (Received 12 February 2015; published 24 February 2015)
}

DOI: 10.1103/PhysRevA.91.029905

PACS number(s): 42.40.-i, 42.30.Wb, 87.59.-e, 99.10.Fg

This paper was published online on 13 January 2015 with incorrect labeling in Fig. 2. The label (b) in the top right panel of Fig. 2 should read as label (d). The figure labeling has been corrected as of 12 February 2015. The figure labeling is incorrect in the printed version of the journal; therefore for the benefit of the print readership, the figure and its caption are replicated below.

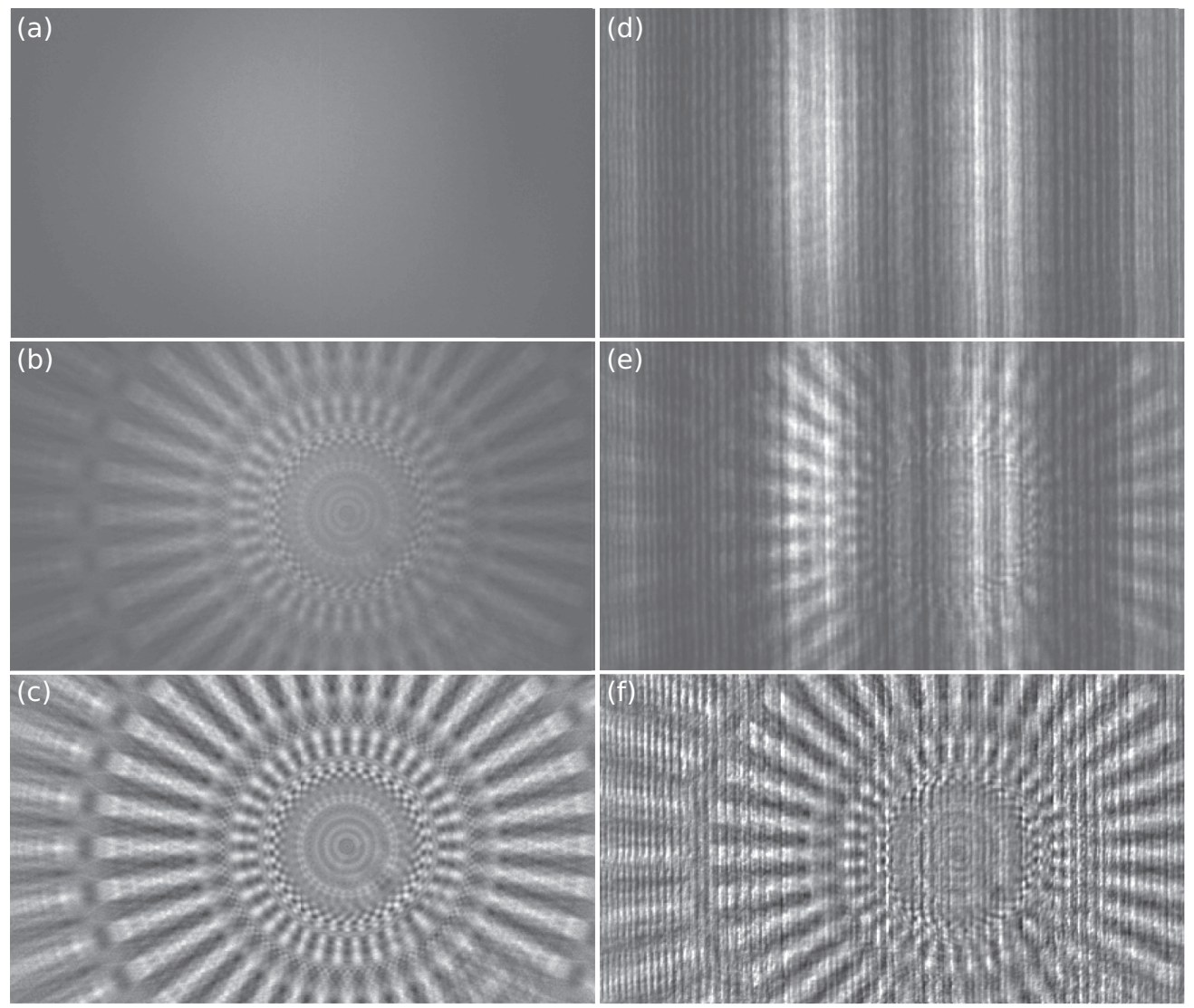

FIG. 2. Illustration of the empty-beam correction for a nearly perfect quasipoint beam illumination provided by a x-ray waveguide (left column), compared to the strongly aberrated case which is deliberately achieved by insertion of a wavefront modifier consisting of W stripes inserted between the waveguide and the object (Siemens star test pattern). Panels (a), (b), and (c) and (d), (e), and (f) show the empty beam, the raw hologram, and the divided image (corrected hologram), respectively. Panel (c) is close to the ideal hologram calculated for ideal spherical or parallel beam (in the two equivalent geometries, as discussed in the text). One pixel corresponds to $25.5 \mathrm{~nm}$ in the object plane.

Published by the American Physical Society under the terms of the Creative Commons Attribution 3.0 License. Further distribution of this work must maintain attribution to the author(s) and the published article's title, journal citation, and DOI. 